\title{
Optimization of Data Processing System for Exercise and Fitness Process Based on Internet of Things
}

\author{
Yiming Wang and Xidan Gong $\mathbb{D}$ \\ Physical Culture Institute, Henan Polytechnic University, Jiaozuo, Henan 454000, China \\ Correspondence should be addressed to Xidan Gong; gxd@hpu.edu.cn
}

Received 8 April 2021; Revised 19 June 2021; Accepted 26 June 2021; Published 7 July 2021

Academic Editor: Wei Wang

Copyright (c) 2021 Yiming Wang and Xidan Gong. This is an open access article distributed under the Creative Commons Attribution License, which permits unrestricted use, distribution, and reproduction in any medium, provided the original work is properly cited.

\begin{abstract}
In the digital network era, people have higher requirements for physical fitness. In the future, physical fitness requires not only good fitness equipment and fitness environment but also more convenient and intelligent health management, service guidance, social entertainment, and other refined fitness services. The innovation of sports and fitness equipment for the digital network era will definitely depend on the development of information technology and network technology. Based on the cutting-edge Internet of Things technology, this thesis focuses on the development and application of a new generation of digital fitness equipment adapted to future development, advocating the new concept of seamless integration of fitness exercise and information services through human-oriented systematic design thinking and providing implementable solutions to realize the science, convenience, and life of public fitness. This thesis uses modern science and technology, especially the Internet of Things (IoT) technology, to fully meet the diversified fitness needs of the fitness crowd as the guide; IoT digital fitness equipment design and application research was newly generated, using a variety of research methods to explore the functional design and application of IoT fitness equipment; the goal is to create a more intelligent and three-dimensional IoT fitness service model in the future. Through the application research of intelligent devices in IoT fitness equipment, the realization of the functions of identity identification, environment perception, and data transmission of IoT fitness equipment is made faster. Intelligent devices can become the interaction channel between fitness service personnel, fitness equipment, and fitness users and also reduce the development cost of IoT fitness equipment. The construction of an IoT fitness cloud service platform and data management system integrates the application of IoT, cloud computing, mobile communication, and other technologies to make IoT fitness service supply remote, real-time, and diversified. While providing convenient and value-added fitness services for fitness people, it also brings sustainable development space for the health service industry.
\end{abstract}

\section{Introduction}

The sports and fitness equipment industry is an important part of the sports industry; at present, fitness equipment product design and development mainly to imitate similar foreign products in the road of independent innovation are slow; coupled with the worldwide fitness equipment product innovation which has not yet appeared, product homogenization is serious and has been difficult to adapt to today's network; information technology and intelligent product design have also become the urgent need to address the fitness equipment industry. This has become a pressing prob- lem for the fitness equipment industry. This thesis is aimed at realizing the application of IoT technology in fitness equipment design, with health as the guide; fully meeting the diversified fitness needs of fitness people as the core; and exploring the application of IoT technology in digital fitness equipment design, not only to provide ideas to overcome the multiple contradictions in the current fitness equipment industry but also to provide the most optimal solution to promote the innovative development of the sports and fitness equipment industry [1]. In modern social life, portable smart devices such as smartphones, tablets, and watches based on digital networked services are widely used, and the integration of 
network-based product application services with smart devices and wearable devices has been more emphasized, with network services more closely connected to real life and the content of services more refined [2].

As people's living standards and health concerns continue to improve, mass fitness is moving in the direction of specialized, scientific, and refined needs. Traditional fitness equipment has a single function, has poor interactivity, lacks guidance, has other drawbacks, and has been difficult to meet the growing diversified fitness needs of people in the era of network information [3]. The innovative development of these fields has provided a more mature environment for the application of IoT technology. The IoT can be extended and expanded to many sports-related fields, especially for the construction of future networked sports and fitness service systems. The digital fitness equipment based on IoT technology proposed in the thesis will play an important role in the future networked fitness service systems, which has high theoretical research significance and practical value [4]. In the digital network era, people also have higher requirements for physical fitness, and the future physical fitness not only needs good fitness equipment and fitness environment but also needs more convenient and intelligent health management, service guidance, social entertainment, and other refined fitness services. The innovation of physical fitness equipment for the digital network era will definitely depend on the development of information technology and network technology [5]. Based on the cutting-edge IoT technology, this dissertation focuses on the development and application of a new generation of digital fitness equipment adapted to future development, advocating the new concept of seamless integration of fitness exercise and information services through human-oriented systematic design thinking and providing implementable solutions to realize the scientific, convenient, and lifelike aspects of public fitness. The research of the thesis actively seizes the historical opportunity of the development of the Internet of Things industry and actively responds to the objective laws and requirements of the development of the Internet of Things industry and the sports and fitness industry, which can both promote the innovative development of the sports and fitness equipment manufacturing industry and help to improve the theoretical research of digital sports and digital fitness Internet of Things [6]. The recommendation system can tap the association between user preferences and information based on the individual differences of each user, such as the basic information of the user and the behavior habits of the user, and help the user find what he or she really needs by actively pushing information to the user. For this reason, it is of great importance and far-reaching significance to successfully introduce recommendation technology into motion monitoring systems to help improve user experience, enhance users' reliance on the system, and build user stickiness, as shown in Figure 1. However, recommendation technology often relies on large-scale data computation to explore the association between users and information, while big data is the product of the current Internet era, and the use of big data processing technology to achieve recommendations has naturally become a new research hotspot, and the sports monitoring system under big data is born under such a demand of the times [7-12].

This thesis focuses on the development and application of IoT fitness equipment, taking the digital fitness treadmill based on IoT as a design case to explore the design concept and ideas of IoT fitness equipment, in order to provide a reference solution for future fitness equipment production and design. By exploring the construction and application of new IoT fitness service modes based on IoT fitness equipment, the modern fitness service mode integrated with IoT, cloud computing, and mobile communication technologies is proposed, which makes the construction of a large fitness service system for the national and even global fitness population possible in the next step. The thesis advocates the fitness equipment design thinking based on the Internet of Things, which can drive fitness equipment, traditional fitness services, and other industries to produce a strong application value, to not only inject more vitality into the development of the emerging fitness equipment industry but also provide reference significance to the adjustment of the entire sports industry structure, which is very important for meeting the masses' scientific fitness needs, improving the market position of the sports and fitness industry, optimizing the industrial structure, and promoting the economy. It is of great social significance to meet the scientific fitness needs of the masses, improve the market position of the sports and fitness industry, optimize the industrial structure, and promote economic transformation. The structure of the whole paper can be described as follows.

In Section 2, we introduce the related work of the IoT technologies and the applications in the related fields. In Section 3, this thesis uses modern science and technology, especially the Internet of Things (IoT) technology, to explore the functional design and application of IoT fitness equipment; the goal is to create a more intelligent and threedimensional IoT fitness service model in the future. In Section 4, through the application research of intelligent devices in IoT fitness equipment, the realization of the functions of identity identification, environment perception, and data transmission of IoT fitness equipment is made faster.

\section{Related Work}

IoT technologies are widely mentioned and applied in the fields of healthcare, transportation, health, logistics, environmental protection, etc. [13-17]. As early as 1995, Bill Gates' book The Way of the Future first proposed the idea of the interconnection of things and things, which was the prototype of IoT. However, this idea was not taken seriously at that time due to the constraints of related technical fields and the lack of products that could prove the prospect of IoT applications. Subsequently, in 1999, Professor Kevin Ashton, director of MIT Auto-ID Center, put forward the more popular concept of Internet of Things (IoT) when studying RFID technology: IoT is based on radio frequency identification technology and equipment, according to the agreed communication protocol and Internet combination, so that the information of items to achieve the Internet of Things is a network formed by relying on radio frequency identification 


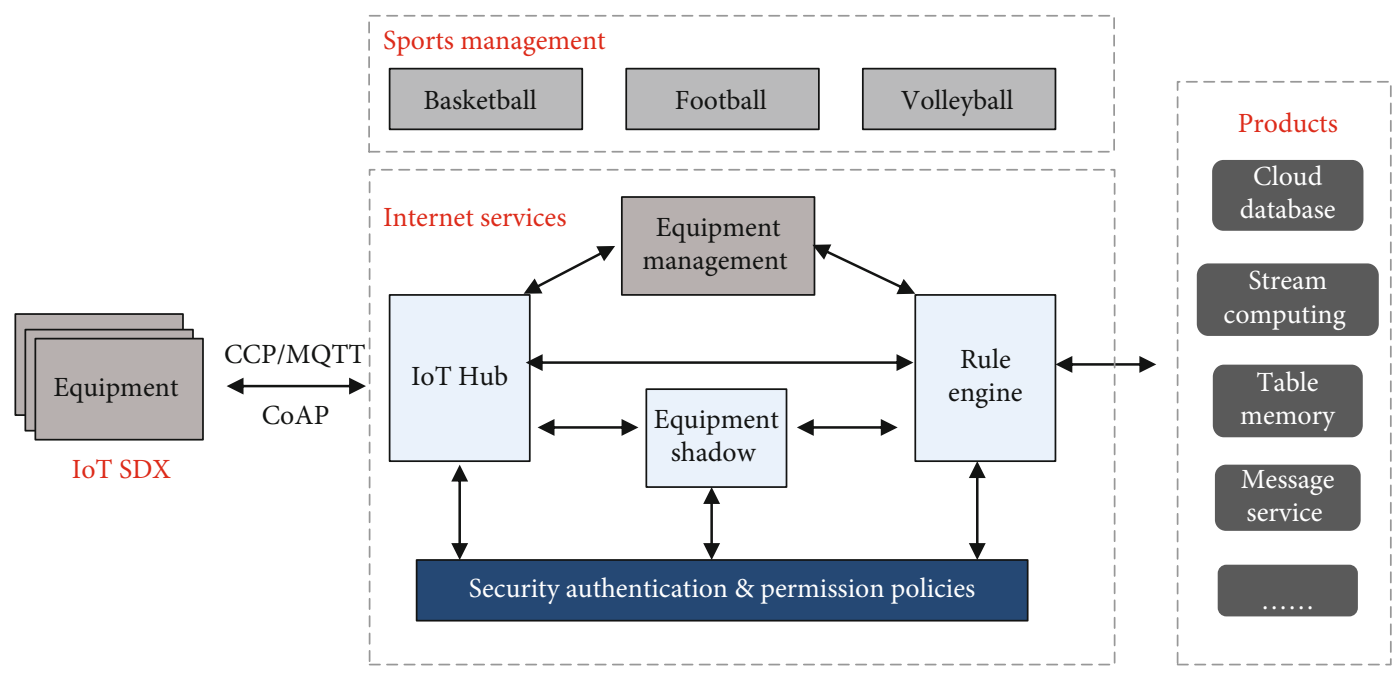

Figure 1: Digital recommendation system based on IoT.

technology and equipment, combining with the Internet according to the agreed communication protocol, so as to realize intelligent identification and management of item information and realize interconnection of item information. Since its focus in the research field is on identification technology, the definition of IoT was not comprehensive enough in the technological context at that time. Subsequently, on November 17, 2005, at the World Summit on the Information Society (WSIS) in Tunis, the International Telecommunication Union (ITU) released the ITU Internet Report 2005: Internet of Things, which formally introduced the more official concept of IoT, which was defined by the reporters as the Internet of Things (IoT) as a network of people to people and people to people by embedding short-range mobile transceivers into various accessories and everyday objects. In September 2009, at the Sino-European Workshop on IoT and Enterprise Environment in Beijing, Dr. Lorent Ferderix, head of the RFID department of the European Commission's Information and Social Media Division, gave the EU the IoT as a dynamic global network infrastructure with selforganizing capabilities based on standards and interoperable communication protocols, where physical and virtual "things" have identity, physical attributes, virtual characteristics, and intelligent interfaces and are seamlessly integrated with information networks. The Internet of Things will constitute the Internet of the future, together with the Internet of Media, the Internet of Services, and the Internet of Enterprises.

In summary, although the concept of IoT is widely proposed, there is no precise and accepted definition of IoT, mainly because IoT is the product of the integration of various technologies including sensor identification, network transmission, data mining, and pervasive computing. It requires the mutual integration of multiple disciplines, and it is closely related to the Internet, mobile communication network, sensor network, etc. Researchers in different fields have different starting points for thinking about IoT, and they cannot reach a consensus in the short term. With the rapid development of mobile Internet and IoT and the introduction of highperformance low-power processing chips, portable measure- ment devices that can collect data of human daily activities in real time have emerged on a large scale, which has greatly promoted the development of related industries in the field of wearable fitness. The Live Pod is a portable exercise apparatus that can monitor the daily activities of a human body in real time. It can collect fitness indicators including the type of fitness movements, exercise status, fitness time, exercise rhythm, energy consumption value per minute of exercise, the ratio and amount of fat and sugar metabolism during exercise, and the capture of exercise form, realizing the real-time sensing of fitness behavior in human daily life [18]. On July 24, 2010, at the China International Fitness Conference held at the China National Convention Center in Beijing, Xia Gao, the CEO of http://YouBody.com, proposed a new concept of fitness Internet of Things (IoT), and the first generation of http://YouBody.com's product was the IoT electronic scale, which implanted a wireless module in the traditional electronic scale and allowed the data related to the electronic scale to be sent to the website and cell phone background [19]. School of Biological and Medical Engineering, Beijing University of Aeronautics and Astronautics, developed an insole-type detection system for quantitative evaluation and early warning of daily behavioral exercise load and health status of motor function, which realizes the sensing and calculation of human exercise load amount and features low cost, low interference, and low energy consumption, further realizing the monitoring management and intelligent guidance of human health.

The well-known brand Nike also uses sensing and computing technology in basketball and training shoes, which has a built-in Nike+ Pressure Force chip that can record users' movement data and then send it to users' mobile devices through wireless data transmission, which can sense users' jump height, confrontation intensity, and reaction speed, and the collected data can help users improve their skills and further guide them to perform healthy exercises [20]. Thanks to the development of the Internet of Things and mobile Internet technologies, technological changes and upgrades are constantly being made in the field of large fitness equipment development and design. The My Wellness 
product launched by Technogym, Italy, combines miniature portable measuring devices with treadmills and strength trainers to realize the monitoring and management of daily life and professional fitness, and fitness data can be shared on the My Wellness platform, while programmed fitness guidance can be obtained [21]. The Iconcept launched by the American Akcome Company takes full advantage of the APP program application of Apple and other smart cell phones to realize the design of treadmill controlled by cell phones, while its cooperation with Google Maps realizes the reproduction of virtual scenes when working out. In addition, there is also the treadmill that can access the Internet launched by Technogym. The treadmill is equipped with a VISIOweb display screen, which can be used for entertainment and leisure while exercising, and at the same time, through the cooperation with social platforms such as Twitter and Facebook, the gym-goers can share and socially entertain themselves while working out [22].

\section{Design of Fitness Equipment Based on IoT Technology}

3.1. Design Objectives. The design goal of IoT fitness equipment is to use IoT technology to connect fitness equipment with professional fitness service resources in real time, to realize the integration of multiple resources, and to provide interactive interfaces for fitness people, social media, digital game developers, fitness service providers, and other resources [23-26]. The IoT fitness equipment collects and transmits personal fitness data in real time through terminal software, realizes massive fitness data storage and processing technology based on IoT mode, provides corresponding fitness service solutions to different fitness people through server-side data analysis and calculation, realizes automatic control of fitness equipment, and finally realizes personalized and customized service modes of fitness service. The main design content of IoT fitness equipment is the IoT hardware system and the corresponding software service system, as shown in Figure 2. The fitness data is obtained and controlled by the whole IoT system from the vessel data and data analysis and finally result evaluation. At present, the hardware system required sensors, automatic control, and network link technology which has been relatively mature; this thesis is mainly based on the IoT fitness equipment hardware required to achieve the function of the main research, to explore the existing technology to achieve the application of IoT fitness equipment; software service system design is based on IoT fitness equipment to achieve the focus of the networked service model.

In the study of the architecture of IoT, the perception layer, as the lowest layer, is the basis for the realization of IoT functions. In the fitness service system based on IoT fitness equipment, the perception layer is mainly to realize fitness equipment identification and intelligent collection of various fitness information, which is composed of various fitness information collection sensors and sensor gateways. Through the IoT fitness equipment, the fitness of the exercise state, exercise environment, life pattern, and other information monitoring are achieved. Fitness data collection is an important function of the perception layer of the IoT fitness service system. The acquisition of user fitness data is also the basis for personalized services; the application of modern sensor technology can not only better achieve the collection of user fitness data but also provide more data interfaces to the relevant fitness service software developers, to facilitate the development of more interesting and practical fitness service software.

The implementation of automatic control can hand over the development of fitness programs and plans to more professional fitness or health service providers, avoiding the damage to fitness users due to blind fitness or operation errors, as shown in Figure 3. For example, the mainstream fitness treadmill in the market will integrate a number of running fitness models; however, many fitness users are not sure which fitness model they should choose to work out; therefore, remote automatic control will be the future fitness equipment development that needs to be paid attention for the function. At the same time, the core of IoT fitness equipment development is also intelligent control; automatic control function is a good reflection of the intelligent level of IoT fitness equipment, which is an important hardware need of IoT fitness equipment. With the development of IoT, cloud computing, wireless mobile communication, and other technologies, the functions of online fitness, remote interaction, and cloud management can be better applied in the design of IoT fitness equipment. IoT treadmill, as the terminal equipment of IoT fitness service mode, will change the "closed" fitness mode of traditional treadmill exercise in the future and realize the quantification, science, and life of fitness, so that the gym-goers can adhere to it for a long time. Through the construction of a relevant IoT fitness service platform, based on the massive fitness data provided by IoT treadmill and other IoT fitness equipment, the quantitative statistics, mining, and analysis of fitness data can be carried out to establish and promote the construction of scientific fitness behavior universal model and provide personalized scientific fitness service for diversified fitness groups.

\subsection{Design of IoT Fitness Equipment Cloud Service Platform.} Through the establishment of the cloud service platform, it can give access to a readily scalable resource pool, while using cloud load balancing technology to deal with the high concurrency of the fitness service system and other issues, and take a dynamic approach to deploy various application systems to maximize the efficiency of the utilization of the fitness service business system resources, so as to meet the IoT fitness service workload and business needs. The specific implementation is as follows.

(1) The core framework of the fitness service system is built based on technologies such as KVM virtualization technology and the cloud platform of Open Stack, which enables efficient processing of business such as large fitness data and fitness services

(2) Methods such as complex systems and complex networks are used to achieve scientific fitness adherence and efficient dissemination mechanisms in the system 


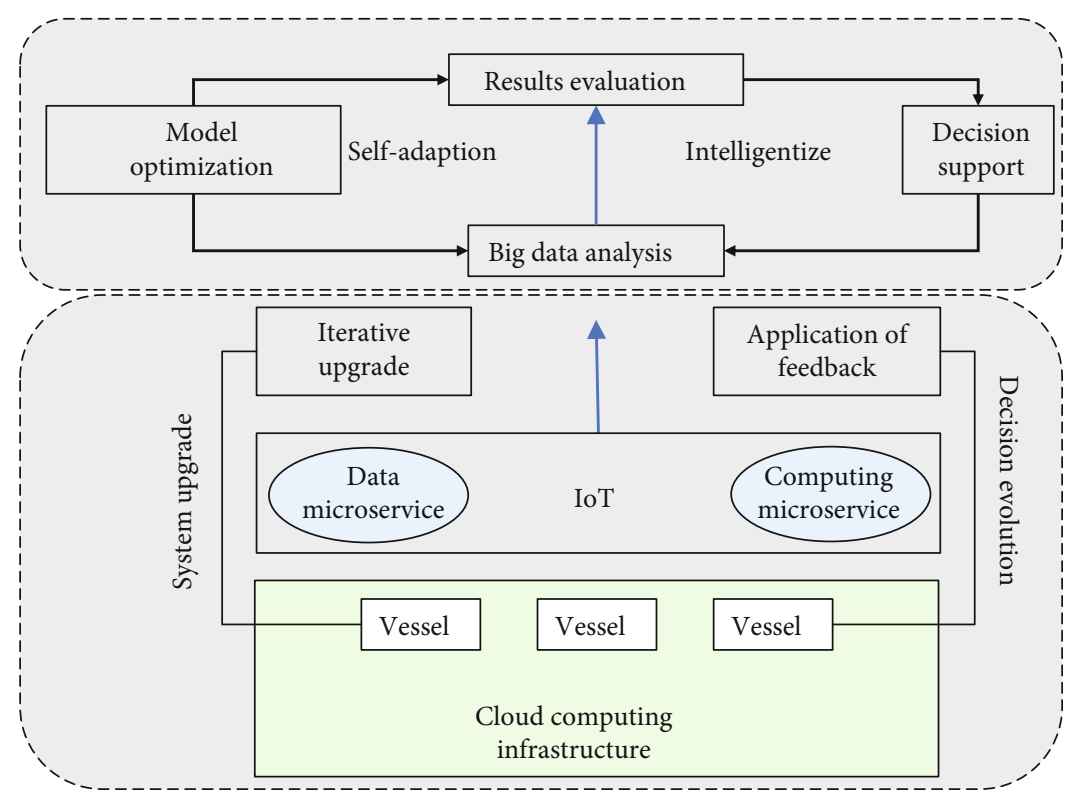

FIGURE 2: IoT fitness equipment application model.

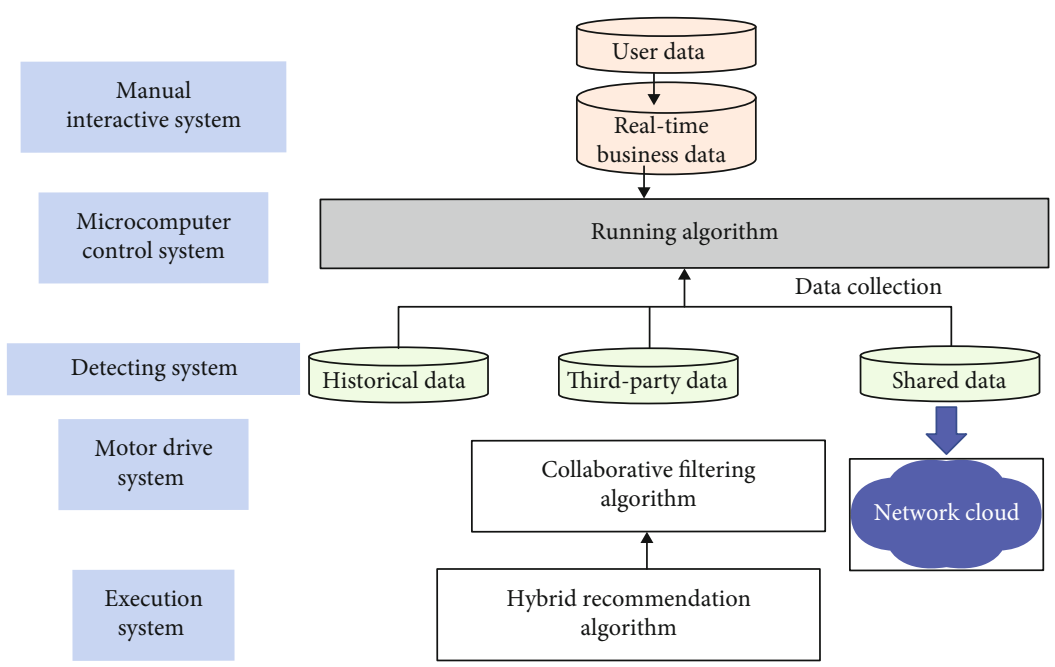

FIgURE 3: Digital treadmill control system structure diagram.

(3) Using REST and other technologies to achieve realtime data transmission between the Android and Apple IOS-based mobile fitness service client APP and the system server, the data is stored in JSON format, and the REST interface is called to realize the server side to parse the data and realize the transmission and interaction of business data

(4) Spring technology is used to control the business logic of massive fitness data in the system

(5) Mongo DB database technology and cloud load balancing technology are used to build the underlying database

The architecture is shown in Figure 4.
Fitness service is based on the soul of the Internet of Things fitness equipment, network services must have the support of server technology, and the server as the center of data processing is the basis of network fitness service provision. The fitness service system based on IoT fitness equipment provides personalized fitness service for fitness users through the collection, analysis, and calculation of fitness user data; in view of the wide area of IoT fitness service, its supporting server needs to have the ability to meet the huge volume, type, multiple sources of data storage, and calculation in real time, and the fitness service application of computing, storage, and network resource relationship is dynamic change. The relationship between computing, storage, and network resources of fitness service applications is dynamic. For the traditional WEB server cluster solution 


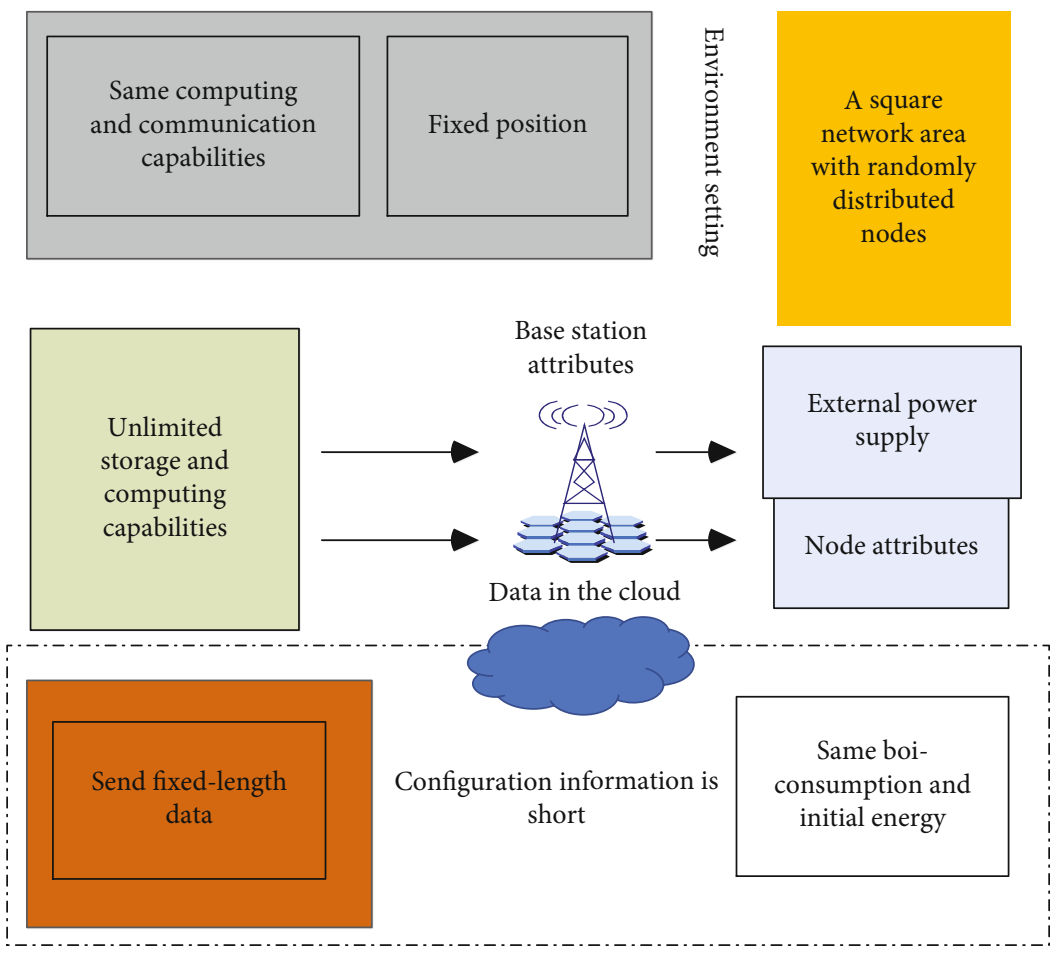

FIGURE 4: Fitness cloud service platform architecture.

model, it is difficult to maintain and support normal operation around the clock; the ordinary form of WEB server and database system can only be used in the early stage; later, with the growth of the service population, it can only rely on the cloud server model to solve this growth crisis. In the process of IoT fitness equipment application implementation, the amount of data from nothing to have, service data from tiny to huge, and its supporting server will certainly experience the upgrade from local to cloud. Therefore, the establishment of a cloud service platform based on IoT fitness equipment is one of the core tasks to realize IoT fitness services. The construction objectives of the IoT fitness cloud service platform are three things.

(1) Create a standard system of IoT fitness equipment. The use of the Internet of Things, cloud computing technology, and Wifi, GPRS, 3G, and other wireless mobile communication network technology to achieve the Internet of Things fitness equipment user physical condition monitoring, remote fitness guidance, and other service functions is an important measure to ensure the development of the scientific nature of the user fitness activities, through the development of data transmission and processing standards to achieve the universal characteristics of the production of IoT fitness equipment

(2) Construct a sharing platform for IoT fitness services, use IoT technology to realize the direct connection between fitness instructors and fitness participants, digitally integrate the fitness-related exercise instruction service information and so on, and send it to IoT fitness equipment users in real time, targeted and remotely through the network. In the conduct of such fitness services, a number of monitoring instruments can be selected for daily fitness, and the exercise status, energy consumption, and life pattern of fitness participants can be monitored in real time in a lifelike manner to provide basic data support for personalized scientific fitness guidance. The cloud fitness service platform can effectively promote the fitness activities of the fitness crowd. Each fitness organization contains fitness service enterprises, social fitness instructors, fitness personal trainers and fitness participants, and other different roles. The construction of a fitness cloud service platform can effectively expand the communication, coordination, supervision, evaluation, and management among organizations, families, and users and finally form an urbanized "network national fitness club" supported by Internet of Things technology

(3) To build an all-weather fitness service system that can work out anytime and anywhere. The cloud fitness service platform is a public service sharing platform based on cloud computing technology serving the public, which should be compatible with different people and different kinds of IoT fitness equipment, integrate various forms and items of fitness terminals, meet the needs of multilevel and different scale fitness guidance systems, and meet the needs of multiprofessional health information service. Its open sharing mode breaks the information island mode of closed and independent traditional fitness network systems 
and adopts cloud computing technology to deploy distributed data centers. It massively reduces the cost of operation, improves the ability to cope with massive data, and expands the scope of system applications. The scattered fitness experts and other resources from different regions and organizations are networked and aggregated using IoT technology to form a large and professional expert fitness service production line, which continuously produces efficient fitness service products and provides them to different regions, thus enabling the public to enjoy the opportunity to receive professional fitness guidance anytime and anywhere and build a 24/7 fitness service system

\subsection{Fitness Process Data Processing and Recommendation} System. Recommendation system evaluation is an important issue in the field of recommendation. It has a variety of evaluation metrics in different application scenarios, and researchers in the industry have not yet formed a unified scientific standard evaluation system, but generally recognized important metrics have been widely used. Among them, recommendation accuracy is the most used evaluation index, i.e., the degree of agreement between the predicted score (or ranking) of the recommendation system and the actual score (or ranking) of the user, which is mainly divided into three categories: prediction accuracy, classification accuracy, and ranking accuracy. The specific calculation formula of the evaluation index is as follows.

Mean absolute error $(M)$ :

$$
M=\lim _{n \longrightarrow \infty} \sum_{i=1}^{n} \frac{\left(p_{i}-r_{i}\right)^{2}}{N} .
$$

Mean squared error $(S)$ :

$$
S(I)=\frac{\lim _{n \longrightarrow \infty} \sum_{i=1}^{n} P_{i}(Q) \cap R_{Q}(I)}{N(x)} .
$$

Root mean square error $(R)$ :

$$
R(I)=\sqrt{\frac{\left|P_{i}(Q) \cap R_{Q}(I)\right|}{U}}, \quad D \leq 1 .
$$

Standard mean absolute error (NMAE):

$$
N(x, y)=\frac{a_{i} \cdot b_{i}(y) \cup b_{i(x)}}{r_{\max }+r_{\min }} .
$$

In the formula, $N$ is the number of objects that have been rated by users, $\left\{p_{i}\right\}$ is the set of predicted user rating values, $\left\{r_{i}\right\}$ is the set of actual user ratings of objects, and $r_{\max }$ and $r_{\text {min }}$ are the maximum and minimum values of user ratings, respectively. The most popular recommendations today are based on proximity relationships, including user-based and object-based recommendation algorithms. However, such recommendation algorithms often determine the association between users or objects by calculating the similarity of users or objects first and recommend objects under similar users or similar objects to the target users, which lacks comprehensive consideration of the target user model and also suffers from the naming conflict problem, i.e., objects of the same nature may have multiple different names, while they are similar in nature, but this type of recommendation algorithms cannot discover and exploit this similarity. To address these problems, model-based collaborative filtering recommendation algorithms have become an inevitable alternative to traditional collaborative filtering recommendation algorithms, among which the traditional matrix factorization (MF) technique is widely used. The following paper will elaborate on the SVD decomposition method in the traditional matrix factorization technique. Singular Value Decomposition (SVD) is a generalization of eigenvalue decomposition to arbitrary matrices. Since the user-object rating matrix may not be a square matrix, this matrix decomposition method can be introduced into the recommendation system to achieve the purpose of reducing the dimensionality of the rating matrix. The method assumes that $R$ is an $M \times N$ matrix, which can be decomposed into the product of three matrices as follows:

$$
R=\lim _{n \longrightarrow \infty} \sum_{i=1}^{n} a_{i} \cdot b_{i}(y),
$$

where $U$ is an $M \times M$ square matrix (the vectors of the square matrix are orthogonal and are called left singular vectors), $\Sigma$ is an $M \times N$ matrix (all elements except those on the diagonal are 0 and arranged in descending order), and $V T$ is an $N \times N$ square matrix (the vectors of the square matrix are orthogonal and are called right singular vectors). First, finding the eigenvalues, one obtains:

$$
\left(R^{T} R\right) p_{i}(x, y)=b \cdot b_{i}(x)
$$

where $v$ is the right singular vector. In addition, it is also obtained that

$$
\begin{aligned}
& \delta_{i}=\sqrt{\alpha_{i}}, \\
& u_{i}=\frac{\sqrt{\alpha_{i}}}{\delta_{i}},
\end{aligned}
$$

where $\delta$ is the singular value and $u$ is the left singular vector. It is known from the existing relevant mathematical knowledge that the value of $\sigma$ decreases rapidly. In other words, the first $W$ singular values arranged from largest to smallest can be taken to describe the matrix approximately, i.e., the matrix $\Sigma$ of $M \times N$ decreases in dimension to $W \times W$, while the square matrix $U$ of $M \times M$ is transformed into $M \times W$ and the square matrix $V T$ of $N \times N$ is transformed into $W$ $\times N$. In this way, the original matrix can be compressed according to a certain proportion and retain the original matrix information, where $W \ll M, W \ll N$, and the degree of reduction of the $R W$ matrix to the original matrix $R$ can be controlled by adjusting the value of $W$. Here, the closer 
$W$ is to $N$, the closer the product of the three matrices on the right-hand side is to the original matrix $R$. Decomposing matrices using the SVD method does not allow the existence of blank parts in the matrix to be decomposed. In fact, it only makes sense to apply the SVD decomposition method to the field of recommendation system after we solve the data sparsity problem. In the recommendation scenario, there are a lot of gaps in the scoring matrix and the data is sparse. For this reason, we need to preprocess the scoring matrix, i.e., fill the missing values in the scoring matrix with the help of a global mean, object scoring mean, or user scoring mean. Of course, some normalization of the matrix is also possible. However, even with the fill strategy, in practical application scenarios, the number of users and items is often in the thousands, and the time complexity of the traditional SVD algorithm $O\left(n^{3}\right)$ is obviously unsolvable in the face of such a large order of magnitude.

\section{System Optimization Test}

A dataset of a certain size is extracted from the file system, and the original file is read using the read function in Python and converted to matrix form by the Data Frame data structure common function under the pandas package. By using the implicit feedback data (user personal attributes, user motion data, and user adoption) and explicit feedback data (user ratings of motion guidance) actually collected by the system for experiments, the root mean square error (RMSE) is used as a performance index to evaluate the recommendation accuracy of the algorithm, so as to investigate the advantages and disadvantages between this algorithm and the SVD decomposition method. Assuming that the difference between users' real ratings and predicted ratings obeys Gaussian distribution, the corresponding loss functions are jointly constructed using the rating matrix $R$ (explicit feedback data) and the user data behavior weighting matrix $W$ (implicit feedback data), and then, the loss functions are optimized by the stochastic gradient descent algorithm (SGD), and the parameter values in the matrix $P$ and matrix $Q$ are calculated and solved using the existing historical rating data, so as to obtain the corresponding matrices $P$ and $Q$, i.e., matrix $P$ is the user hidden class matrix, which represents the user's preferences, and matrix $Q$ is the motion guidance hidden class matrix, which represents the features of motion guidance. Since the datasets extracted from the HDFS file system in this experiment are .txt files, which need some conversion processing, and the method of machine learning is adopted, a large number of third-party learning libraries need to be used, so the Python programming language is chosen here for implementation. The following is the specific experimental procedure.

In the first step, a dataset of a certain size is extracted from the HDFS file system, and the original file is read using the read function in Python and converted from .txt format to .csv format and then converted to matrix form by the Data Frame data structure common function under the pandas package. The user score table (score_data Frame) contains table id, user id, campaign guide id, and score value, and some of the data are shown in Figure 5.

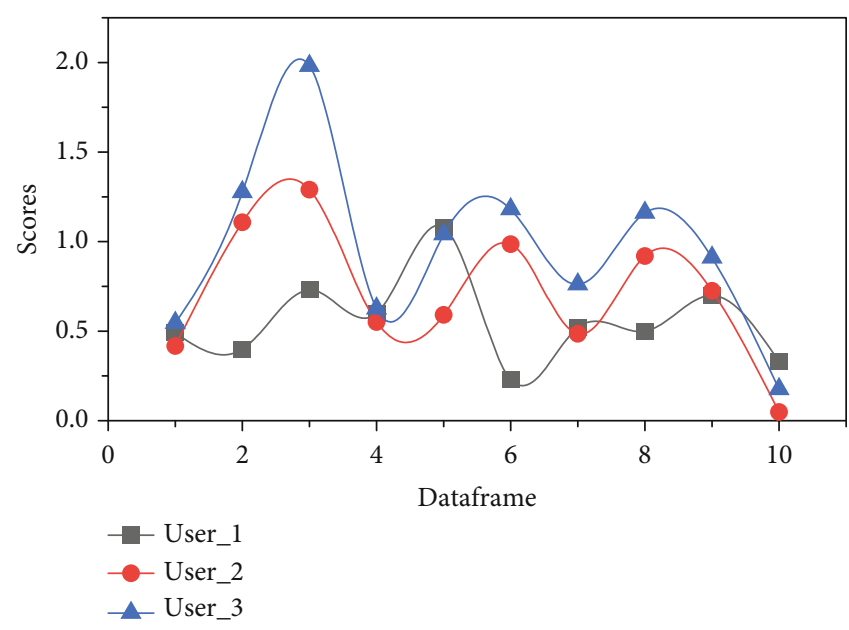

Figure 5: Sports guide rating chart.

In the second step, we calculate the weights of user data behaviors (user's personal information, personal movement data collected by user wearing the bracelet, and behavioral data such as operating APP) to generate the weighting matrix $W$ of user data behaviors and then combine with the scoring matrix $R$ to construct the corresponding loss function. In the third step, the stochastic gradient descent algorithm (SGD) is used to optimize the loss function by finding the partial derivatives of the parameters $p(u, f)$ and $q(f, i)$ to determine the fastest direction of descent, and then, iterative computation keeps optimizing the parameters until the parameters converge. Here, the parameters converge at about 500 iterations, and the iteration ends with the output of two low-dimensional matrices $P$ and $Q$, as shown in Figure 6 .

The experimental results in this paper use the root mean square error (RMSE) as a performance indicator to evaluate the algorithm recommendation accuracy, and the calculation formula is shown above, i.e., the smaller the value, the higher the algorithm recommendation accuracy. The RMSE of the weighted matrix decomposition recommendation algorithm based on the hidden semantic model is calculated to be 0.12, and the RMSE of the SVD decomposition method is calculated to be 0.42 . It can also be seen from Figure 7 that the two fine curves match well, and the coarse curve deviates to a greater extent, indicating that the recommendation accuracy of the improved recommendation algorithm is higher, i.e., the recommendation accuracy of the weighted matrix decomposition recommendation algorithm based on the hidden semantic model is better than that of the traditional SVD decomposition method. The reason for this difference is that the singular value matrix decomposition model first needs to mean fill the historical rating data and then perform matrix decomposition to obtain the correlation submatrices, which reduce the model credibility and naturally result in lower recommendation accuracy. On the contrary, the Latent Factor Model (LFM) fully utilizes the user item rating matrix as the explicit feedback data and introduces the idea of weighting to build a weighted matrix of user data behaviors as the implicit feedback data, which deeply explores the user's preferences from both explicit and implicit aspects, making 


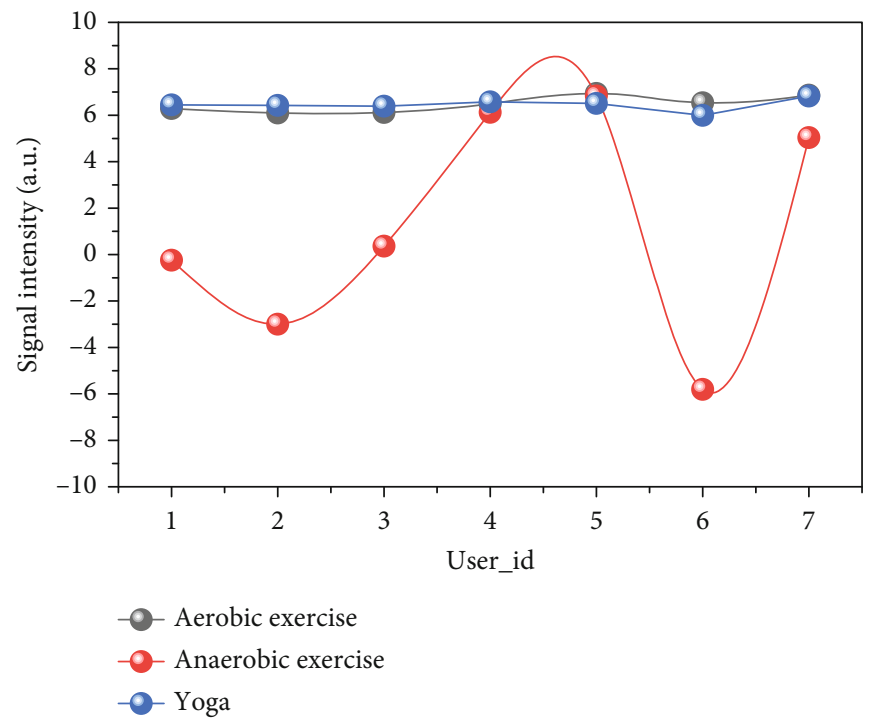

FIgURe 6: User hidden class matrix.

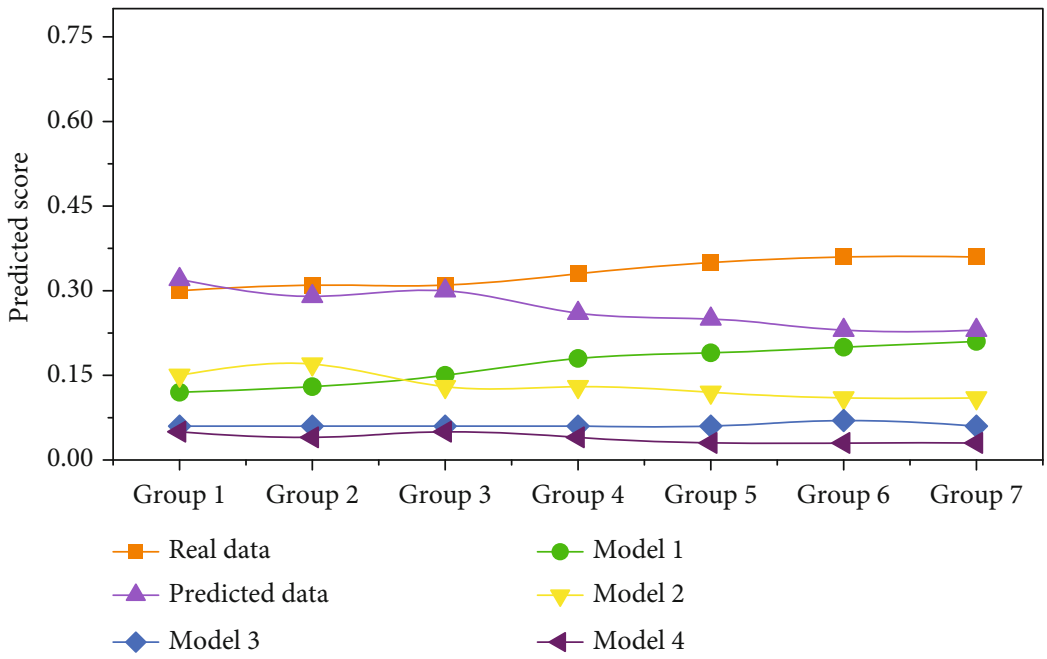

FIGURE 7: Algorithm predicted score vs. actual score trend.

the improved recommendation algorithm more perfect and the recommendation accuracy higher.

After users use the system for a period of time, the system backend can calculate the corresponding rating value based on the ratio of the user's browsing time for a certain exercise guide to the browsing time for all the exercise guides that have been browsed, which eventually forms the user rating table, i.e., the user-generated display rating data. At the same time, the implicit behavioral data generated by users, such as personal information and sports data mentioned above, are jointly extracted from the server My SQL database to HBase by Sqoop tool. Then, the offline batch computing framework of Map Reduce is used to decompose the user rating matrix into user implicit class matrix and motion guidance implicit class matrix to generate the original recommendation model. Finally, the Spark Streaming streaming computing framework is used to combine the user data behavior weighting matrix generated by the current user behavior data (implicit data), so as to reupdate the user rating matrix (i.e., user pre- diction rating matrix) and update the recommendation model to get the online recommendation results, and Figure 8 shows the exercise guidance recommendation. The popularity of IoT fitness equipment will strongly contribute to the application and development of related IoT fitness services. From the perspective of practitioners, the growing sports and fitness service industry has given rise to a large demand for sports industry practitioners, and the frontline work of fitness service product providers, that is, fitness service industry practitioners with professional fitness guidance and management knowledge, has made up for a large labor demand gap. More sports instructors, personal trainers, and students from sports colleges and universities are attracted to enter the sports and fitness service industry, and the proportion of the number of people employed in the sports and fitness service industry to the number of people employed in society as a whole will increase significantly. The improvement of the quantity and quality of sports practitioners will play an important role in promoting the 


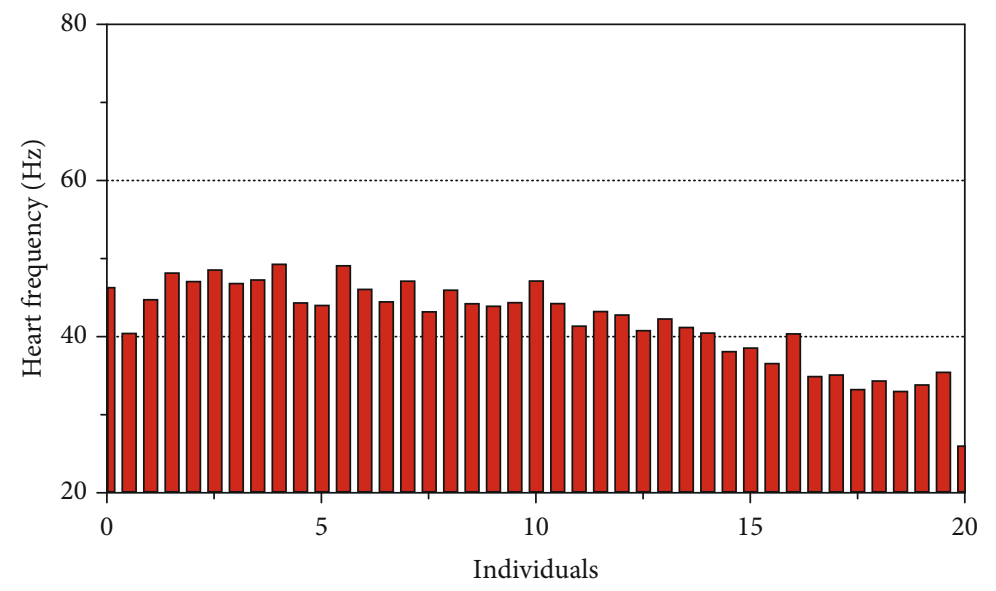

Figure 8: Exercise instruction frequencies.

industrialization of fitness services, improving the quality and level of fitness services, increasing the added value of fitness industry products, promoting the overall improvement of the sports industry, enhancing the core competitiveness of the fitness service industry, and promoting the sustainable development of the fitness industry.

\section{Conclusion}

The case design of IoT digital treadmill not only takes into account the advantages of traditional digital treadmill but also makes use of sensor technology, embedded technology, and automatic sensing technology to make IoT treadmill a fashionable and modern fitness tool, which provides an important basis for the hardware design of other types of IoT fitness equipment. Through the application research of intelligent devices in IoT fitness equipment, it makes the realization of identity identification, environment perception, data transmission, and other functions of IoT fitness equipment faster. Smart devices can become the interaction channel between fitness service personnel, fitness equipment, and fitness users and also reduce the development cost of IoT fitness equipment. The construction of an IoT fitness cloud service platform and data management system integrates the application of IoT, cloud computing, mobile communication, and other technologies to make IoT fitness service supply remote, real-time, and diversified. While providing convenient and value-added fitness services for fitness people, it also brings sustainable development space for the health service industry. The exercise monitoring system researched and implemented in this paper is set in the application context of outdoor sports parks, where there is a situation of large-scale user use, so improvements are made to the multitask realtime scheduling mechanism of the system on the outdoor smart Bluetooth base station.

\section{Data Availability}

The data used to support the findings of this study are available from the corresponding author upon request.

\section{Conflicts of Interest}

The authors declare that they have no known competing financial interests or personal relationships that could have appeared to influence the work reported in this paper.

\section{Acknowledgments}

The study was funded by the University-level Humanities and Social Science Research Fund of Henan Polytechnic University in 2021: Research on the Influence of China Tennis Tour on the Operation Mode of Chinese Traditional Tennis Matches from the Perspective of Self-created IP (number: SKND2021-22).

\section{References}

[1] M. Måløy, F. Måløy, R. Lahoz-Beltrá, J. C. Nuño, and A. Bru, "An extended Moran process that captures the struggle for fitness," Mathematical Biosciences, vol. 308, pp. 81-104, 2019.

[2] G. R. Tomkinson, K. D. Carver, F. Atkinson et al., "European normative values for physical fitness in children and adolescents aged 9-17 years: results from 2779165 Eurofit performances representing 30 countries," British Journal of Sports Medicine, vol. 52, no. 22, pp. 1445-1456, 2018.

[3] G. R. Tomkinson, J. J. Lang, and M. S. Tremblay, “Temporal trends in the cardiorespiratory fitness of children and adolescents representing 19 high-income and upper middle-income countries between 1981 and 2014," British Journal of Sports Medicine, vol. 53, no. 8, pp. 478-486, 2019.

[4] Z. Guo and X. Yan, "Fitness partition-based multi-objective differential evolutionary algorithm and its application to the sodium gluconate fermentation process," Chemometrics and Intelligent Laboratory Systems, vol. 177, pp. 8-16, 2018.

[5] E. Tikkanen, S. Gustafsson, and E. Ingelsson, "Associations of fitness, physical activity, strength, and genetic risk with cardiovascular Disease," Circulation, vol. 137, no. 24, pp. 2583-2591, 2018.

[6] J. M. Abdullah and T. Ahmed, "Fitness dependent optimizer: inspired by the bee swarming reproductive process," IEEE Access, vol. 7, pp. 43473-43486, 2019. 
[7] B. Foroughi, M. Iranmanesh, H. F. Gholipour, and S. S. Hyun, "Examining relationships among process quality, outcome quality, delight, satisfaction and behavioural intentions in fitness centres in Malaysia," International Journal of Sports Marketing \& Sponsorship, vol. 20, no. 3, pp. 374-389, 2019.

[8] E. Foxall and N. Lanchier, "Generalized stacked contact process with variable host fitness," Journal of Applied Probability, vol. 57, no. 1, pp. 97-121, 2020.

[9] N. Watanabe, S. S. Sawada, K. Shimada et al., "Relationship between cardiorespiratory fitness and non-high-density lipoprotein cholesterol: a cohort study," Journal of Atherosclerosis and Thrombosis, vol. 25, no. 12, pp. 1196-1205, 2018.

[10] J. M. Scott, E. C. Zabor, E. Schwitzer et al., "Efficacy of exercise therapy on cardiorespiratory fitness in patients with cancer: a systematic review and meta-analysis," Journal of Clinical Oncology, vol. 36, no. 22, pp. 2297-2305, 2018.

[11] M.-S. Shin and H. Lee, "Effect of additional firing process after sintering of monolithic zirconia crown on marginal and internal fitness," The Journal of Korean Academy of Prosthodontics, vol. 57, no. 4, pp. 321-327, 2019.

[12] T. Tarumi and R. Zhang, "Cerebral blood flow in normal aging adults: cardiovascular determinants, clinical implications, and aerobic fitness," Journal of Neurochemistry, vol. 144, no. 5, pp. 595-608, 2018.

[13] X. Liu, Q. Pan, and M. He, "Stochastic dynamics in the fitnessbased process which can be on behalf of the standard Moran, local and Wright-Fisher processes," Journal of Theoretical Biology, vol. 460, pp. 79-87, 2019.

[14] K. A. G. Kremling, S.-Y. Chen, M.-H. Su et al., "Dysregulation of expression correlates with rare-allele burden and fitness loss in maize," Nature, vol. 555, no. 7697, pp. 520-523, 2018.

[15] H. B. D. Silva, L. K. Beura, H. Wang et al., "The purinergic receptor P2rx7 directs metabolic fitness of long-lived memory CD8+ T cells," Nature, vol. 559, no. 7713, pp. 264-268, 2018.

[16] E. Dean, "The fitness to practise process demystified: what every nurse and manager need to know," Nursing Management, vol. 27, no. 5, pp. 9-11, 2020.

[17] H. Zbinden-Foncea, M. Francaux, L. Deldicque, and J. A. Hawley, "Does high cardiorespiratory fitness confer some protection against proinflammatory responses after infection by SARS-CoV-2?," Obesity, vol. 28, no. 8, pp. 1378-1381, 2020.

[18] B. V. Adkar, S. Bhattacharyya, A. I. Gilson, W. Zhang, and E. I. Shakhnovich, "Substrate inhibition imposes fitness penalty at high protein stability," Proceedings of the National Academy of Sciences of the United States of America, vol. 116, no. 23, pp. 11265-11274, 2019.

[19] L. Feng, A. S. Raman, M. C. Hibberd et al., "Identifying determinants of bacterial fitness in a model of human gut microbial succession," Proceedings of the National Academy of Sciences of the United States of America, vol. 117, no. 5, pp. 2622-2633, 2020.

[20] J. C. Jones, P. N. Q. Pascua, T. P. Fabrizio et al., "Influenza A and $\mathrm{B}$ viruses with reduced baloxavir susceptibility display attenuated in vitro fitness but retain ferret transmissibility," Proceedings of the National Academy of Sciences of the United States of America, vol. 117, no. 15, pp. 8593-8601, 2020.

[21] G. Taino, E. Oddone, G. Corona, R. Foti, and M. Imbriani, "The fitness to work certificate in a worker exposed to ionizing radiation with an oncological disease: criteria and assessment process," Radioprotection, vol. 54, no. 4, pp. 303-307, 2019.
[22] C. J. Watson, A. L. Papula, G. Y. P. Poon et al., "The evolutionary dynamics and fitness landscape of clonal hematopoiesis," Science, vol. 367, no. 6485, pp. 1449-1454, 2020.

[23] W. Wei, M. Guizani, S. H. Ahmed, and C. Zhu, "Guest editorial: special section on integration of big data and artificial intelligence for Internet of Things," IEEE Transactions on Industrial Informatics, vol. 16, no. 4, pp. 2562-2565, 2020.

[24] W. Wang, N. Kumar, J. Chen et al., "Realizing the potential of the Internet of Things for smart tourism with $5 \mathrm{G}$ and AI," IEEE Network, vol. 34, no. 6, pp. 295-301, 2020.

[25] J. Han, N. Lin, J. Ruan, X. Wang, W. Wei, and H. Lu, “A model for joint planning of production and distribution of fresh produce in agricultural Internet of Things," IEEE Internet of Things Journal, vol. 8, no. 12, pp. 9683-9696, 2021.

[26] S. H. Ahmed, V. H. C. de Albuquerque, and W. Wei, "Guest editorial: special section on advanced deep learning algorithms for industrial Internet of Things," IEEE Transactions on Industrial Informatics, vol. 17, no. 4, pp. 2764-2766, 2021. 